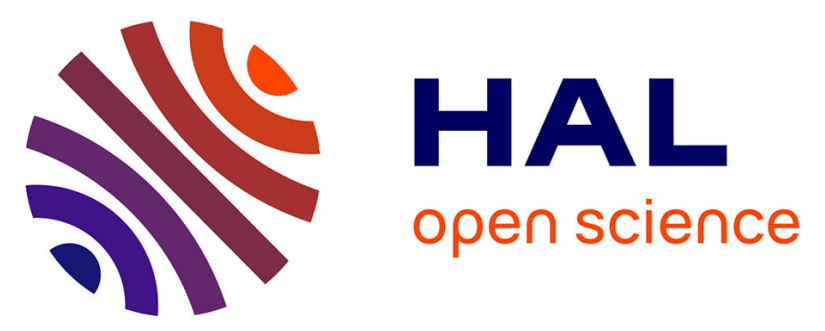

\title{
Morphological brain plasticity induced by musical expertise is accompanied by modulation of functional connectivity at rest.
}

Baptiste Fauvel, Mathilde Groussard, Gaël Chételat, Marine Fouquet, Brigitte Landeau, Francis Eustache, Béatrice Desgranges, Hervé Platel

\section{To cite this version:}

Baptiste Fauvel, Mathilde Groussard, Gaël Chételat, Marine Fouquet, Brigitte Landeau, et al.. Morphological brain plasticity induced by musical expertise is accompanied by modulation of functional connectivity at rest.. NeuroImage, 2014, 90, pp.179-188. 10.1016/j.neuroimage.2013.12.065 . inserm00939280

\section{HAL Id: inserm-00939280 https://www.hal.inserm.fr/inserm-00939280}

Submitted on 30 Jan 2014

HAL is a multi-disciplinary open access archive for the deposit and dissemination of scientific research documents, whether they are published or not. The documents may come from teaching and research institutions in France or abroad, or from public or private research centers.
L'archive ouverte pluridisciplinaire HAL, est destinée au dépôt et à la diffusion de documents scientifiques de niveau recherche, publiés ou non, émanant des établissements d'enseignement et de recherche français ou étrangers, des laboratoires publics ou privés. 


\section{Elsevier Editorial System(tm) for NeuroImage \\ Manuscript Draft}

Manuscript Number: NIMG-13-1913R2

Title: Morphological cerebral plasticity induced by musical expertise is accompanied by modulation in functional connectivity at rest.

Article Type: Regular Article

Section/Category: Cognitive Neuroscience

Corresponding Author: Pr Hervé Platel, Ph.D

Corresponding Author's Institution: Université de Caen

First Author: Baptiste FAUVEL, doctorate student

Order of Authors: Baptiste FAUVEL, doctorate student; Hervé PLATEL, Professor; Mathilde

GROUSSARD, doctor; Gaël CHETELAT, Professor; Marine Fouquet, doctor; Brigitte Landeau; Francis

EUSTACHE, Professor; Béatrice Desgranges, doctor

Abstract: The aim of this study was to explore whether musical practice-related gray matter increases in brain regions are accompanied by modifications in their resting-state functional connectivity. 16 young musically experienced adults and 17 matched nonmusicians underwent an anatomical magnetic resonance imaging (MRI) and a resting-state functional MRI (rsfMRI). A whole-brain two-sample t test run on the T1-weighted structural images revealed four clusters exhibiting significant increases in gray matter (GM) volume in the musician group, located within the right posterior and middle cingulate gyrus, left superior temporal gyrus and right inferior orbitofrontal gyrus. Each cluster was used as a seed region to generate and compare whole-brain resting-state functional connectivity maps. The two clusters within the cingulate gyrus exhibited greater connectivity for musicians with the right prefrontal cortex and left temporal pole, which play a role in autobiographical and semantic memory, respectively. The cluster in the left superior temporal gyrus displayed enhanced connectivity with several language-related areas (e.g., left premotor cortex, bilateral supramarginal gyri). Finally, the cluster in the right inferior frontal gyrus displayed more synchronous activity at rest with claustrum, areas thought to play a role in binding sensory and motor information. We interpreted these findings as the consequence of repeated collaborative use in general networks supporting some of the memory, perceptual-motor and emotional features of musical practice. 
December $26^{\text {th }} 2014$

Dear Editor,

Thank you very much for providing us the opportunity to revise this paper. We also greatly thank the two reviewers for their constructive and valuable comments. Hereafter are our answers to each comment and remark. A new version of the manuscript has also been transmitted. We hope that these last modifications will give you all satisfaction and that our work is now suitable for a publication in NeuroImage.

Sincerely,

H. Platel 
- Musical practice shapes the brain structurally, mainly in auditory and motor areas.

- Musical practice is also accompanied by functional plasticity.

- We linked these two forms of brain plasticity using seed-based approach of rsfMRI.

- Only musicians show more gray matter volume and enhanced functional connectivity.

- Musical practice modulates brain networks implicated in high cognitive functions. 


\section{${ }^{*} 6$. Response to Reviews}

Reviewer \#1: The authors addressed the concerns raised in the previous review. I only have a few more comments.

- Page 10: I appreciate that the authors have made the effort to show the significant correlations also as bar graphs. They are informative. However, since these represent values from the statistical maps selected based on significance thresholds, additional statistical tests on these values are inappropriate (also called "double dipping") and it should be clearly stated that these bar graphs are for visualization purposes only. Thank you for pointing this, we have added the precision that these bar graphs are not supplementary analysis but for description purpose only: "Moreover, for descriptive purpose, the means of their correlation coefficient values for each group are represented in a bar graph (Fig. 3) that also shows whether these means were significantly different from zero $\left({ }^{*} p \leq 0.05,{ }^{*} p \leq 0.01, * \star \star p \leq 0.001\right) . "$

- According to the new analysis with seed area volume as covariate, group differences for three of the four seed areas are no longer significant according to the previously set statistical threshold for the image analysis. This does not invalidate the previous findings of significant differences, but strongly suggests that seed volume is an important factor that might explain some of the group differences in the functional data. I would not call this "almost unchanged" (P 12). We have removed the formulation "almost unchanged" and rephrased the sentence in order to clearly state that the results were lowered in their statistical power when seed's volume are controlled as covariates : "

Moreover, supplementary analysis showed that results remained significant, but lowered in their statistical power when local GM volumes were statistically controlled for. This suggests that local GM volumes partially influenced the functional results, but that the increases we found regarding restingstate functional connectivity in the musically-experienced group cannot however wholly be explained by their higher GM volume."

- Page 12: "Additionally, results showed that each of these four areas displayed enhancements of resting state functional connectivity with one or several regions of the brain, but solely in the musician group (see figure 2)." This seems to imply that only musicians show positive correlations and should be reworded to reflect that musicians showed increased correlations compared to the nonmusicians.

We acknowledge that "in the musicians group only" was misleading. We have replaced it with "in the musically experienced group compared with the nonmusicians group" and have added clearly that for each analyses, the reverse comparisons (testing nonmusicians VS musicians)lead to no significant result: "results showed that each of these four areas displayed higher resting-state functional connectivity with one or several regions of the brain in the musically experienced group than in the nonmusician group (see Fig. 2). Interestingly, none of the analyses revealed an area that was more functionally connected in the nonmusician group compared with the musically experienced one." Thank you.

- P5: "undoubtedly in response to" should be replaced by "probably related to"

Thank you for this advice, it is done in the new version of the manuscript: "For their part, the expert musicians (Luo et al. 2012) exhibited stronger resting-state time-series correlations among the perceptual and motor networks, probably related to the regular multimodal activity described earlier."

- Manuscript should be proof-read by a native speaker of English. Some examples are: P4 "maintaining associated the activity of brain areas", P. 5 "individuals owning an expertise", P7 "uncomfortably installation", P 9 "cluster whom size were upper than 15 voxels" etc.

The new version of the manuscript has now been entirely proof-red and corrected by a native speaker of English. We hope the writing to be now proper. 
- P6: "enhancement of gray matter partly reflecting the dentritic growth" should be changed to " volume increase of gray matter, possibly related to dendritic growth" or similar, since the association has not been established in humans.

Thank you for prompting us to be more careful when translating findings from animals studies to human. We have reworded this sentence : "with practicerelated enhancement of GM possibly related to the dendritic growth required for building or strengthening synaptic projections".

- P6: It is unclear what "read music in an automated manner" means. We agree that the term "automatic" was fuzzy, it meant that musicians of this study knew music theory and were able to read scores skillfully enough to practice music and to learn new pieces without the help of a teacher anymore... We have replaced the former formulation with "They were recruited from the local conservatory or other classical music schools where they have learned music theory for at least 7 years, in order to be able to read music and to master their musical instrument in an autonomous manner."

- P6: It should be specified in the manuscript if musicians were music students or if they studied other subjects and merely practiced musical instruments in their spare time.

Thank you for asking us to add this precision, musicians of this study were studying other topics and played music as a leisure activity. It is now clearly stated in the manuscript : "Sixteen were students who currently played a musical instrument (violin, cello, guitar, flute, recorder, trumpet, clarinet, or piano) in their spare time at least several times a week, and had been doing so for a mean duration ( \pm SD) of 16.13 ( \pm 4.29 ) years at the time of the study."

- P9: I assume that several t-tests were conducted comparing the two groups' maps for each of the seeds rather than only one test.

You assume right, this error has been corrected.

- P11: In the description of the group comparison results (3.2.1 through 3.2.4), it should always be clear that musicians were compared to nonmusicians. Statements with "more ..." should be specified with "than xy". In most cases, it will be sufficient to add "than nonmusicians". We have revised this part of the manuscript to always clearly indicate that the musicians' group was compared with the nonmusicians one.

Reviewer \#2: In general, the Discussion is now much better and stronger what comes to the arguments for the findings. Also, the new Figure 3 is informative showing that also nonmusicians have significant connectivity and that there are some connections that are significant only in musicians. Here are some minor corrections I would recommend to be done:

- At the end of the section 1.4, the benefits of your choice of this type of approach could be briefly stated (also serving as a connector to the following chapter, which is the aims)

We have added a short sentence, which, according to us, briefly summarizes the benefits of our method : "In the case of expertise studies, because rsfMRI does not require the performance of any particular task, functional connectivity changes may reflect a cumulative effect of experience over time (Lewis et al. 2009), and can therefore be used as complementary to task-related fMRI for highlighting practice-related functional brain changes in dismissing the potential confounds due to differences in behavioral performances."

- In section 1.5, the second "either" seems to be unnecessary in the 3 rd sentence

We have corrected this error thank you.

- 2.1 Remove extra parentheses in the sentence "The remaining seventeen.." This error has been corrected, thank you. 
- the last sentence in the Discussion (According to us..) is somewhat complicated and probably unnecessary. Also I consider the most important message is stated in the "In addition..." sentence. I recommend to conclude to that.

Thank you have for this recommendation, the new version of the manuscript now concludes with : "In addition, it also shows that these morphological particularities are accompanied by modulations in their resting-state functional connectivity with many other areas of the brain, ranging from subcortical regions to high-level cognitive areas, via the primary sensorimotor cortex." The former last part of the Discussion has been moved just before. - While the GM volume may not explain the findings, what are the alternative explanations? There are not so much about those, even a short listing of alternative explanations would be enough hint for the future studies.

We have putted more emphasis on several plausible suggestions regarding alternative explanations of our findings, and what should be done in future works on this topic : "In future works, it could be worthwhile taking other proxies into account, such as the frequency of training and the number of musical pieces recently learned, in view of the hypothesized role of resting-brain neural activity in consolidating new learning, as well as in maintaining synchronous the activity of areas that are frequently jointly engaged during active states (Fox and Raichle, 2007). Moreover, adding diffusion tensor imaging (DTI) data could help to clarify the link between morphological and resting-state functional modulation of the brain in the framework of expertise-related plasticity given that resting-state functional connectivity also reflects the brain's anatomical connectivity (van den Heuvel et al. 2009)."

- Native English language check could be appropriate for the final version The new version of the manuscript has now been entirely proof-read and corrected by a native speaker of English. We hope the writing to be now proper. 
Morphological brain plasticity induced by musical expertise is accompanied by modulation of functional connectivity at rest

Baptiste Fauvel $^{a b c d}$, Mathilde Groussard ${ }^{a b c d}$, Gaël Chételat ${ }^{a b c d}$, Marine Fouquet ${ }^{a b c d}$, Brigitte Landeau $^{a b c d}$, Francis Eustache ${ }^{a b c d}$, Béatrice Desgranges ${ }^{a b c d}$, and Hervé Platel ${ }^{a b c d}$

a INSERM, U1077, Caen, France

${ }^{\mathrm{b}}$ Université de Caen Basse-Normandie, UMR-S1077, Caen, France

${ }^{\mathrm{c}}$ Ecole Pratique des Hautes Etudes, UMR-S1077, Caen, France

${ }^{\mathrm{d}}$ Caen University Hospital, U1077, Caen, France

Correspondence and reprint requests:

Hervé Platel, Inserm - EPHE-Université de Caen/Basse-Normandie, Unité U1077, U.F.R de Psychologie, Université de Caen/Basse-Normandie, Esplanade de la Paix, 14032 Caen Cedex, France

Phone: +33 (0)2 315665 91; Fax: +33 (0)2 315666 93, e-mail: herve.platel@unicaen.fr 
Abstract The aim of this study was to explore whether musical practice-related gray matter increases in brain regions are accompanied by modifications in their resting-state functional connectivity. 16 young musically experienced adults and 17 matched nonmusicians underwent an anatomical magnetic resonance imaging (MRI) and a resting-state functional MRI (rsfMRI). A whole-brain two-sample $t$ test run on the T1-weighted structural images revealed four clusters exhibiting significant increases in gray matter (GM) volume in the musician group, located within the right posterior and middle cingulate gyrus, left superior temporal gyrus and right inferior orbitofrontal gyrus. Each cluster was used as a seed region to generate and compare whole-brain resting-state functional connectivity maps. The two clusters within the cingulate gyrus exhibited greater connectivity for musicians with the right prefrontal cortex and left temporal pole, which play a role in autobiographical and semantic memory, respectively. The cluster in the left superior temporal gyrus displayed enhanced connectivity with several language-related areas (e.g., left premotor cortex, bilateral supramarginal gyri). Finally, the cluster in the right inferior frontal gyrus displayed more synchronous activity at rest with claustrum, areas thought to play a role in binding sensory and motor information. We interpreted these findings as the consequence of repeated collaborative use in general networks supporting some of the memory, perceptual-motor and emotional features of musical practice.

Keywords: brain plasticity, musical practice, voxel-based morphometry (VBM), rsfMRI 


\section{Introduction}

\subsection{Musical practice as a model for studying brain plasticity.}

Musical expertise has become a useful model for investigating practice-related brain plasticity in humans (Dawson 2011; Fauvel et al. 2013). Performing music is a complex behavior, which relies on immediate and accurate associations between motor sequences and auditory events. Neuroimaging studies have shown that musical training leads to structural plasticity within auditory and motor areas (Münte et al. 2002), as well as a reinforced functional coupling of these regions during musical tasks (Haueisen and Knösche 2001; Bangert et al. 2006). Moreover, repeated musical practice improves not only perception and action, but also general cognitive processes such as memory (short- and long-term), executive functions and attention (Pantev and Herholz 2011). The reason is that, in addition to engaging specific brain areas, the cognitive processes applied to musical material involve broad networks that are used to process other kinds of stimuli as well (Gaab and Schlaug 2003; Bialystok and DePape 2009). This is especially true for experienced musicians, who have to master increasingly difficult pieces of music (Pallesen et al. 2010). Finally, brain plasticity related to musical practice has also been observed in several white-matter (WM) tracts such as the corpus callosum (Schlaug et al. 1995; Öztürk et al. 2002; Bengtsson et al. 2005), the corticospinal tract (Bengtsson et al. 2005; Imfeld et al. 2009) and the arcuate fasciculus (Bengtsson et al. 2005).

Thus, musical practice seems to be both a multimodal and a multiprocess activity that involves large parts of the brain.

\subsection{Learning-related modulation of resting-state functional connectivity.}

Researchers have recently discovered that during wakeful rest, the brain exhibits spontaneous activity, believed to be related to neuronal firing rather than to simple physiological noise (heart rate and breathing; Van den Heuvel and Hulshoff Pol 2010). With the use of resting-state functional connectivity, it seems that the pattern of correlations between the blood-oxygen-level dependent (BOLD) time series of several distinct brain areas points to the existence of a networks organization (Damoiseaux et al. 2006) similar to those known to be engaged during the performance of sensorimotor and cognitive tasks (Van den Heuvel and Hulshoff Pol 2010) and dependent upon the brain's anatomical connectivity (Van den Heuvel et al. 2009). Additionally, some authors have suggested that recent experiences and learning episodes can modulate subsequent patterns of resting-state functional connectivity both within and between the networks they recruited. For example, Lewis et al. 
(2009) found that sensory areas activated during an intensive training session for a very demanding visual task subsequently exhibited increased resting-state functional connectivity within parietal and frontal regions known to be involved in the control of spatial attention. Also using a visual learning paradigm, Urner et al. (2013) highlighted both short- and longlasting learning-related changes in resting-state functional connectivity between the hippocampus and striatum. Regarding the motor domain, Vahdat et al. (2011) found that motor training reinforces resting-state functional connectivity within both a motor and a somatosensory network, while Albert et al. (2009) reported modulations in resting-state functional connectivity in the wake of new complex motor learning, but not after simple, familiar motor activities. This argues in favor of a learning consolidation function of restingstate brain activity. These findings have been replicated with studies featuring more cognitive tasks and with an emphasis on behavioral performances. Veroude et al. (2010) showed Dutch participants a weather report in Mandarin Chinese and assessed their resting-state functional connectivity both before and after an incidental auditory recognition task. When they divided the sample into high and low learners, they found that the high learners displayed not only greater post-task reorganization of resting-state functional connectivity between the regions involved in phonological shape storage (the auditory forms representation of words), but also stronger pre-task resting-state functional connectivity between the regions that sustain phonological rehearsal. These results pointed to both pre-existing and learning-induced differences between the two groups. Finally, a piece of evidence in favor of a memory consolidation role of task-related modulation of resting-state functional connectivity comes from a study by Tambini et al. (2010). They observed enhanced resting-state functional connectivity between the hippocampus and a portion of the lateral occipital lobe following incidental episodic encoding requiring highly associative memory processes for later recall (objects and faces), but not after episodic encoding with relatively low levels of subsequent associative memory (scenes and faces). Interestingly, the coefficient of correlation between the time series of the hippocampus and lateral occipital lobe during the post-encoding resting session was predictive of retrieval performances, thus powerfully demonstrating the importance of task-related modulation of resting-state functional connectivity for learning.

Taken together, the aforementioned studies have given rise to the idea that, even in the absence of external stimuli or demands, the brain is constantly sharing information, consolidating recent learning, and maintaining associated the activity of brain areas that are likely to be used together in the future (Fox and Raichle 2007). 


\subsection{Expertise-related modulation of resting-state functional connectivity.}

Several studies have specifically investigated the effects of long-term expertise on the architecture of resting-state functional connectivity. One of them took Chinese chess masters as model of experts (Duan et al. 2012), and another choose professional or student musicians (Luo et al. 2012). In accordance with these different types of expertise, Duan et al. (2012) focused on cognitive resting networks, whereas Luo et al. (2012) looked at perceptual and motor networks. The boardgame experts in the first study (Duan et al. 2012) displayed a particular pattern of the default mode network (DMN; a set of areas more active at rest than during task performance, thought to sustain spontaneous mind wandering, and mainly comprising the posterior cingulate cortex and prefrontal cortex, as well as temporal and parietal areas Damoiseaux et al. 2006) that included the caudate nucleus. This structure is not classically viewed as belonging to the DMN, but is involved in motivation and decisionmaking processes, and was found to be strongly engaged in the expert players during a chess task. For their part, the expert musicians (Luo et al. 2012) exhibited stronger resting-state time-series correlations among the perceptual and motor networks, probably related to the regular multimodal activity described earlier. These rsfMRI studies of long-term expertise suggest that changes in resting-state functional connectivity can be observed in individuals owning an expertise.

\subsection{Morphometric differences-based resting-state functional connectivity.}

One method of investigating the brain's organization at rest is to look for the overall pattern of connectivity between the BOLD signal time courses of one specific area (classically called seed area) and those of the rest of the brain. This area is chosen because it has been shown to be of relevance for the population and/or cognitive process being studied. Several works have already combined structural MRI and rsfMRI by choosing the structurally affected areas for subsequent resting-state functional connectivity analysis. This has been done in the framework of several pathologies: heroin dependence (Yuan et al. 2010), antipsychotic-naive first-episode schizophrenia (Lui et al. 2009), idiopathic generalized epilepsy (Wang et al. 2012), social anxiety disorder (Liao et al. 2011); and expertise: professional badminton players (Di et al. 2012) and baduk (go) players (Jung et al. 2013). In the case of expertise studies, because rsfMRI does not require the performance of any particular task, functional connectivity changes may reflect a cumulative effect of experience over time (Lewis et al. 2009), and can therefore be used as complementary to task-related 
fMRI for highlighting practice-related functional brain changes in dismissing the potential confounds due to differences in behavioral performances.

\subsection{Aims of the present study.}

In sum, musical practice shapes the brain both structurally and functionally, with practice-related enhancement of GM possibly related to the dendritic growth required for building or strengthening synaptic projections (Draganski and May 2008; Anderson 2011). There is sometimes an overlap between expertise-related structural and functional plasticity (Ilg et al. 2008; Groussard et al. 2010b). Furthermore, resting-state functional connectivity between regions could either reflect their anatomical connectivity (WM fiber tracts), their amount of concomitant activation during active states, or recent learning consolidation. We therefore reasoned that musical practice-related structural plasticity could be accompanied by the modulation of resting-state functional connectivity. Accordingly, we generated restingstate functional connectivity maps from brain areas that displayed greater GM volume in a sample of experienced musicians than in a group of nonmusician counterparts, and ran a second between-group comparison to highlight areas that had significantly increased restingstate functional connectivity with the structurally modified regions in the musician group. Furthermore, we conducted a supplementary analysis to investigate the relationship between our structural and functional results.

\section{Material and Methods}

\subsection{Participants.}

Thirty-three young volunteers (13 men, mean age $\pm S D: 24.35 \pm 3.39$ years, mean educational level $\pm S D$ : $15 \pm 1.56$ years of schooling) with no history of neurological or psychiatric disease took part in this study (Table 1). Sixteen were students who currently played a musical instrument (violin, cello, guitar, flute, recorder, trumpet, clarinet, or piano) in their spare time at least several times a week, and had been doing so for a mean duration $( \pm$ $S D)$ of $16.13( \pm 4.29)$ years at the time of the study. They were recruited from the local conservatory or other classical music schools where they have learned music theory for at least 7 years, in order to be able to read music and to master their musical instrument in an autonomous manner. The remaining seventeen students were strictly nonmusicians (none had ever taken part in musical performances or received music lessons, except for the weekly hour 
of basic musical education in high school; they were common listeners). Participants stated that they had no hearing deficits and did not possess absolute pitch. This study was approved by the regional ethics committee (Comité de Protection des Personnes Nord Ouest III), and informed written consent was obtained from all participants.

\subsection{MRI data acquisition.}

All the participants underwent a single MRI session composed of structural (T1, T2) and functional sequences (non-EPI T2*and rsfMRI) that lasted approximately 45 minutes (T1 = 10'; T2 = 5'; hippocampal sequence $=8^{\prime} ;$ FLAIR $=6$ '; T2 ${ }^{*}=4^{\prime} ;$ rest $=12^{\prime}$ ').

All the images were acquired using the Philips (Eindhoven, The Netherlands) Achieva 3.0T scanner at the CYCERON center (Caen, France). For each participant, a high-resolution T1-weighted structural image was acquired using a 3D fast-field echo sequence (3D-T1-FFE sagittal, time of repetition $(\mathrm{TR})=20 \mathrm{~ms}$, time of echo $(\mathrm{TE})=4.6 \mathrm{~ms}$, flip angle $=20^{\circ}, 170$ slices, slice thickness $=1 \mathrm{~mm}$, no gap, field of view $(\mathrm{FOV})=256 \times 256 \mathrm{~mm}^{2}$, matrix $=256 \mathrm{x}$ 256 , in-plane resolution $=1 \times 1 \mathrm{~mm}^{2}$, acquisition time $\left.=9.41 \mathrm{~min}\right)$, followed by a highresolution T2-weighted spin echo anatomical image (2D-T2-SE sagittal) and a non-echo planar imaging (EPI) T2* image (2D-T2*-FFE axial, sensitivity encoding (SENSE) factor $=$ $2, \mathrm{TR}=3505 \mathrm{~ms}, \mathrm{TE}=30 \mathrm{~ms}$, flip angle $=90^{\circ}, 70$ slices, slice thickness $=2 \mathrm{~mm}, \mathrm{FOV}=256$ x $256 \mathrm{~mm}^{2}$, matrix $=128 \times 128$, acquisition voxel size $=2 \times 2 \times 2 \mathrm{~mm}^{3}$ ).

Resting-state functional images were acquired using an interleaved 2D T2* SENSE EPI sequence designed to reduce geometrical distortions by using parallel imaging, a shorter echo time, and smaller voxels (2D-T2*-FFE-EPI axial, SENSE factor $=2$, TR $=2382 \mathrm{~ms}$, TE $=30 \mathrm{~ms}$, flip angle $=80^{\circ}, 42$ slices, slice thickness $=2.8 \mathrm{~mm}$, no gap, in-plane resolution $=$ $2.8 \times 2.8 \mathrm{~mm}^{2}, 280$ volumes, acquisition time $\left.=11.5 \mathrm{~min}\right)$. The first six volumes were discarded due to saturation effects. Participants were equipped with earplugs and the scanner room's light was turned off. During the scan, which was the last one of the MRI scanning session, participants were asked to relax, lie still in the scanner and keep their eyes closed, without actually falling asleep. A subsequent debriefing questionnaire allowed us to especially check that the participants had indeed easily stayed awake throughout the duration of the rsfMRI scan and that nothing particular had disturbed their attention during the scanning session (e.g., MRI noise, uncomfortable position, feeling hungry). 


\subsection{Data preprocessing and statistical analysis.}

\subsubsection{Demographic statistical analyses.}

Two-sample $t$ tests were run on the demographic data to confirm that age and educational level did not differ significantly between the two groups (age: $t(2,31)=-0.49, p=$ 0.62 ; educational level: $t(2,31)=-0.4, p=0.69)$. These demographic information are provided in Table 1.

\subsubsection{Anatomical data preprocessing.}

All imaging data processing were performed using statistical parametric mapping software (SPM5; Wellcome Trust Centre for Neuroimaging, London, UK) implemented in Matlab 7.4.

The T1-weighted structural data for each participant were spatially normalized to the Montreal Neurological Institute (MNI) template and segmented to isolate the GM partitions using the voxel-based morphometry (VBM5) toolbox (Ashburner and Friston 2005). These GM partitions were modulated to correct for nonlinear warping only, so that the values in the resulting images would feature volumes corrected for brain size. Then, the modulated images were smoothed using an 8-mm full width at half maximum (FWHM) isotropic Gaussian kernel. Finally a binary GM mask was created prior to the statistical analysis to include only those voxels having the highest probability of belonging to GM. The binary mask was created by first calculating the mean GM, WM, and cerebrospinal fluid (CSF) images of all subjects, and then, by setting those voxels of GM to 1 where $(\mathrm{GM}>\mathrm{WM}) \cap(\mathrm{GM}>\mathrm{CSF}) \cap(\mathrm{GM}>$ $0.3)$.

\subsubsection{Functional data preprocessing.}

The rsfMRI data were preprocessed using a procedure already described elsewhere (Mevel et al. 2012). They were first checked for artefacts through the application of the TSDiffAna routines (http://imaging.mrccbu.cam.ac.uk/imaging/DataDiagnostics), during which a variance volume was created for each participant to ensure that most of the signal variability was restricted to the cortex. None of the 33 participants showed evidence of significant movements (> $3 \mathrm{~mm}$ translation or $1.5^{\circ}$ rotation) or abnormal variance distribution. For each participant's dataset, the EPI volumes were corrected for slice timing and realigned on the first volume. Data were then spatially normalized using a technique designed to reduce geometric distortion effects (Villain et al. 2010). Briefly, for each individual, this procedure 
includes (1) the coregistration of the mean EPI volume, non-EPI T2*, T2 and T1 volumes, (2) the warping of the mean EPI volume to match the non-EPI T2* volume, (3) the segmentation of the T1 volume using the VBM 5.1 'Segment' procedure with the International Consortium for Brain Mapping (ICBM) / MNI priors, (4) the normalization of the coregistered T1, EPI, and non-EPI T2* volumes using the parameters issued from the T1 segmentation, and (5) the 4-mm FWHM smoothing of the EPI volumes.

Finally, another binary GM mask was created from the group's segmented mean GM T1 volume in conjunction with the mean non-EPI-T2* volume in the MNI space (including only voxels with values above 0.25 in both mean images). This GM mask was used in all the statistical analyses of the functional imaging data described below.

\subsubsection{Anatomical imaging analysis / Seeds determination}

First, a two-sample $t$ test was run on the whole GM volume to assess differences between the two groups of participants. Results were viewed as significant at $p$ (unc) $\leq 0.001$ and we only considered clusters whom size were upper than 15 voxels $(\mathrm{k} \geq 15)$.

Based on the results of this anatomical MRI data analysis, clusters where the GM volume was significantly higher in the musicians than in the nonmusicians were taken as the seeds for the subsequent functional connectivity analyses. To this end, images resulting from the anatomical analysis were binarized and the clusters were isolated and converted into a .mat file using the MarsBaR toolbox (Brett et al. 2002).

\subsubsection{Functional imaging analyses.}

The resting-state time series of the seeds were extracted using the MarsBaR toolbox (Brett et al. 2002), and correlated with the resting-state time series of all the other voxels included in the GM mask analysis. The six head motion parameters were controlled as nuisance variables, and we also applied a high-pass filter in order to remove the lowfrequency component of the fMRI data time series. The resulting $r$ correlation coefficients were then converted into a $z$ score by Fisher's $r$-to- $z$ transformation to improve the Gaussian normality of the distribution. Since the two datasets (rsfMRI and aMRI) did not have the same original spatial resolution, a differential Gaussian kernel smoothing of 6.9 mm FWHM was applied to the functional connectivity map. In this manner, we obtained an equivalent data smoothing of 8 mm FWHM (final rsfMRI smoothing ${ }^{2}=\sqrt{ }\left(\right.$ initial aMRI smoothing ${ }^{2}$ - voxel size $^{2}$; Poline et al. 1995). Finally we ran two-sample $t$ tests on the musicians' and nonmusicians' maps to pinpoint between-group differences in the functional connectivity of 
each seed. Only results that were significant at an uncorrected $p \leq 0.001$ and for a cluster size upper than 15 voxels were considered. The significant clusters resulting from these second analyses are referred to as target areas below.

\subsubsection{Supplementary analysis investigating the link between} anatomical and functional imaging results.

A complementary analysis was carried out in order to investigate the link between the structural and functional results. In other words, we wanted to address whether the effects observed on functional connectivity in musically experienced partcicipants would be explained by the higher GM volume observed in the seed areas. To this aim, we replicated the four between-groups comparisons of the functional connectivity maps in taking the GM volume of the respective seed areas as covariates.

\section{Results}

The means of the resting-state functional connectivity maps generated from the anatomical results are displayed in Figure $1(p(\mathrm{fwe}) \leq 0.0001, k=0)$ for both groups.

All the significant clusters resulting from the between-groups analysis are listed in Table $2(p$ unc) $\leq 0.001, k=15)$ and displayed in Figure $2(p($ unc $) \leq 0.005)$. Moreover, for descriptive purpose, the means of their correlation coefficient values for each group are represented in a bar graph (Fig. 3) that also shows whether these means were significantly different from zero $(* p \leq 0.05, * * p \leq 0.01, * * * p \leq 0.001)$.

\subsection{Effects of musical expertise on whole-brain gray-matter volume.}

In order to see whether one of the two groups had significantly higher GM volume in the brain, a two-sample $t$ test was performed on the segregated GM volume data. The musicians > nonmusicians contrast yielded four clusters: right posterior cingulate gyrus; right middle cingulate gyrus; left superior temporal gyrus; and right inferior orbitofrontal gyrus. The opposite contrast testing nonmusicians > musicians did not reveal any significant voxels.

\subsection{Effects of musical expertise on functional connectivity between the} seeds and the whole brain.

In order to reveal if any of the four seed areas with enhanced GM volume in the musician group also had greater resting-state functional connectivity with other brain regions, we generated whole-brain resting-state functional connectivity maps for the two groups and 
ran two-sample $t$ tests on them. For each of the four seeds, these analyses highlighted one or more areas with more strongly correlated resting-state functional activity in the musician group, whereas the reverse comparison (nonmusicians > musicians) failed to show up any differences.

\subsubsection{Seed 1: right posterior cingulate gyrus.}

The two-sample $t$ test we used to compare the groups' functional connectivity maps for the first seed (cluster within the right posterior cingulate gyrus) revealed a significantly stronger correlation in the musically experienced group than in the nonmusician one between the resting-state time series activity of this area and those of voxels located in the right inferior orbitofrontal gyrus, extending to the anterior cingulate cortex.

\subsubsection{Seed 2: right middle cingulate gyrus.}

The two-sample $t$ test comparing the functional connectivity maps generated from the second seed (within the right middle cingulate gyrus) showed that this region had more functional connectivity at rest with the left superior temporal pole and right inferior frontal gyrus in the musically experienced group compared to the nonmusician group.

\subsubsection{Seed 3: left superior temporal gyrus.}

The comparison of the resting-state functional connectivity maps for the third seed (within the left superior temporal gyrus) highlighted several significant clusters when comparing the musically experienced group to the nonmusician group. In the left hemisphere, the analysis revealed enhanced functional connectivity at rest with the premotor cortex, middle anterior temporal gyrus and middle frontal gyrus. We also found enhanced connectivity with the globus pallidus in the right hemisphere and, bilaterally, with the inferior part of the precentral gyrus and the supramarginal gyrus.

\subsubsection{Seed 4: right inferior orbitofrontal gyrus.}

The last two-sample $t$ test run on the functional connectivity maps for the fourth seed (within the right inferior orbitofrontal gyrus) showed that the resting-state time series of the claustrum (bilaterally) was more synchronous with that of this seed in the musically experienced group than in the nonmusician group. 
3.3 Replication of the functional connectivity map comparisons with seeds' GM volumes as covariates.

The replications of the group comparisons for the four functional connectivity maps with their corresponding seed's GM volume as covariate resulted in lowering the significance of the differences (that now survived at $p \leq 0.005$ ) for the first (corresponding seed: right posterior cingulate gyrus), second (corresponding seed: right middle cingulate gyrus) and fourth networks (corresponding seed: right inferior orbitofrontal gyrus). The result remains almost unchanged with the initial significance threshold $(p \leq 0.001)$ for the third network (corresponding seed: left superior temporal gyrus).

\section{Discussion}

In order to extend our knowledge about the brain plasticity triggered by musical practice, we designed the present study to find out whether the enhancement of regional GM volume observed in a group of experienced musicians is associated with changes in their functional connectivity at rest. To this end, we used structurally enhanced areas found in the musically experienced participants group to generate resting-state functional connectivity maps, which we then compared with those of nonmusicians.

Results showed that the experienced musician group had significantly more GM volume within the right posterior and middle cingulate cortex, left primary auditory cortex, and right inferior frontal gyrus. Structural plasticity of the left primary auditory cortex induced by musical practice has been established cross-sectionally several times (Schneider et al. 2002; Gaser and Schlaug 2003), and a GM increase within the cingulate gyrus (especially in its posterior part) has been attested in children after 15 months of musical training (Hyde et al. 2009). Concerning the right inferior frontal gyrus, a recent cross-sectional study showed that its GM density rises with the amount of musical expertise (James et al. 2013). Additionally, results showed that each of these four areas displayed higher resting-state functional connectivity with one or several regions of the brain in the musically experienced group than in the nonmusician group (see Fig. 2). Interestingly, none of the analyses revealed an area that was more functionally connected in the nonmusician group compared with the musically experienced one. Moreover, supplementary analysis showed that results remained significant, but lowered in their statistical power when local GM volumes were statistically controlled for. This suggests that local GM volumes partially influenced the functional results, but that the increases we found regarding resting-state functional connectivity in the musically-experienced group cannot however wholly be explained by their higher GM 
volume. Finally, as shown in Figure 3, almost all the brain areas we found to be more functionally connected at rest in the musically experienced group compared with the nonmusicians one were also significantly connected in the nonmusician group (compared with the null hypothesis). This suggests that the reinforced functional connectivity we found in the musically trained subjects was more of a quantitative nature rather than a qualitative one. In other words, musical skills would rather rest upon already existing general networks instead of on specific ones, which would be newly created especially for this activity.

On the basis of the literature, in the following sections, we speculate about the relevance of our results in the framework of musical practice and described four functional networks.

\subsection{An autobiographical related network.}

The posterior cingulate gyrus was more functionally connected to the right inferior orbitofrontal gyrus in the musically experienced group. The posterior cingulate gyrus and the prefrontal cortex are important parts of the DMN (Damoiseaux et al. 2006), and during active state, they are the core system of the autobiographical memory network (Martinelli et al. 2012), with the posterior cingulate gyrus sustaining episodic self-related memory (Maddock et al. 2001), and the orbitofrontal gyrus its emotional component (Kross 2009; Etkin et al. 2011). Interestingly, music is widely assumed to trigger both emotion and autobiographical memories, engaging the posterior cingulate cortex (Ford et al. 2011). In an fMRI study, Groussard et al. (2010b) showed that when expert musicians had to judge the familiarity of melodies they had learned to play in the past, they activated the posterior cingulate and orbitofrontal cortex more strongly than nonmusicians, suggesting that these familiar musical pieces were linked to autobiographical events (the various autobiographical contexts of learning and performing).

We could therefore propose that the higher resting-state functional coupling between the right posterior cingulate gyrus and the right inferior orbitofrontal gyrus in musicallytrained participants is triggered by the frequent association of general memory processes with emotional stimuli (music) while learning and recalling pieces of music (Groussard et al. 2010b). 


\subsection{A semantic related network.}

The right middle cingulate gyrus is more functionally connected at rest with the left temporal pole and the right inferior frontal gyrus. These three regions belong to the restingstate salience network, which is implied for the evaluation of external and internal stimuli and the monitoring of errors (Seeley et al. 2007). Moreover, during active state, the middle cingulate gyrus is an important structure for working memory processes, especially the detection of incongruity, salience and/or surprising events (Etkin et al. 2011), whereas on the contrary, the inferior frontal gyrus seems to process the regular rules that govern both language and Western tonal music (Tillman et al. 2003). Finally, the temporal pole is viewed as the region where semantic knowledge about unique entities are stored (Simmons and Martin 2009; Ross and Olson 2011).

Interestingly, the middle cingulate gyrus has been shown to be more highly activated in musicians than in nonmusicians in fMRI studies of auditory working memory for musical sounds (Pallesen et al. 2010) and musical semantic memory (Groussard et al. 2010a). Moreover, both the right inferior frontal gyrus (Groussard et al. 2010) and the left temporal pole (Platel et al. 2003) have been found to be involved in semantic memory tasks using musical material.

Taking these findings together, we can hypothesize that the higher resting-state functional connectivity observed between these three areas could be induced by frequent access to musical semantic knowledge and auditory structures, and their maintenance in working memory.

\subsection{A language related network.}

The left superior temporal gyrus (Brodmann area, BA 48) corresponds to the primary auditory cortex, which contains auditory frequency-selective neurons that code for sound perception and production (Price 2012).

In our study, the musicians exhibited greater functional connectivity between this region and subcortical area (right globus pallidus), bilateral motor areas (inferior part of the precentral gyrus) and the left premotor cortex, the bilateral supramarginal gyrus, an associative auditory area (left middle anterior temporal gyrus), and the left middle frontal gyrus. It is interesting to note that all these regions could belong to the resting-state auditory network and that during active state, all of them (except for the globus pallidus) have been linked to one or several stages of language perception, comprehension and/or production (Price 2012). 
It would not be very surprising that musical practice leads to a strengthening of a language-related network, as more and more authors argue that the brain regions classically dedicated to speech are actually involved in all forms of auditory-motor behavior, including musical practice (Dick et al. 2010).

For instance, works have shown a functional coupling of primary motor and auditory areas during the perception and production of these two behaviors (speech: Watkins et al. 2003; music: Haueisen and Knösche 2001; Bangert et al. 2006). Moreover, secondary auditory and motor areas have been shown to be active during the mental imagery of speech (Shergill et al. 2002) and music (Kristeva et al. 2003). Then, the role of the supramarginal gyrus in the sight reading of words (Stoeckel et al. 2009) has also been demonstrated by Sergent et al. (1992) for musical scores, and the left middle dorsolateral frontal gyrus seems to play a role in attributing verbal labels to music notes (Bermudez and Zatorre, 2005). Finally, the globus pallidus is a subcortical motor pathway of the brain which has been found to be involved in visually guided, voluntary, small-amplitude movements in animal studies (Alamy et al. 1995), its function in a musical practice context could therefore be link with the subtle motor modulation that is required to ensure accurate synchrony.

Taking these findings together, we suggest that the greater resting-state functional connectivity observed in the musically experienced group among these areas dedicated to language perception and production processes could be explained by the activity of regularly reading musical scores and generating musical phrases.

\subsection{An emotional related network.}

The final area that showed a higher GM volume in musicians was located within the right inferior orbitofrontal gyrus, and was more functionally connected at rest with the bilateral claustrum. Together, these three regions form part of the resting-state executive control network (Damoiseaux et al. 2006).

During active state, the orbitofrontal gyrus is involved in decision making and emotional state regulation (Hariri et al. 2000; Phan et al. 2002; Payer et al. 2012), and has often been found to be active during neuroimaging protocols that require the processing of emotion in facial expressions (Nakamura et al. 1999), prosody (Morris et al. 1999; George et al. 2012) and music (Brattico et al. 2011). The claustrum is a subcortical area that contains retinotopic and somatosensory maps that code for bodily and visceral states by owning inputs and outputs with almost the entire cortex (Crick and Koch 2005). This allows for the integration of external and internal information. In a recent paper, Smythies et al. (2012) 
extended this view of the claustrum's function to all voluntary behavior. They likened the claustrum to a general of war who "could review the whole situation at a glance, and make instant decisions about what orders to send back".

It seems coherent to assume that these three regions play a collaborative role in musical practice, integrating the emotion aroused by the sounds into the modulated planning of future motor sequences in order to maintain the proper expressiveness of the piece being played.

\section{Conclusion}

To sum-up, after identifying four regions with increased GM volume in the musician group, we looked for brain areas whose resting-state time-series activity was more closely synchronized with one of these regions in the musician group than in the nonmusician group. For each of the four comparisons, analyses revealed a network of one or more areas, allowing us to supply integrative interpretations of their hypothetical cognitive functions during musical practice.

The first network we found comprised autobiographical memory-related regions belonging to the DMN. In the context of musical practice, we suggest that this network is recruited by the encoding, storage and recall of melodies that are partly based on their emotional and autobiographical contextual components. The second network we identified comprised areas that belong to the salience network. Its involvement during musical practice could be explained by the need to access to semantic musical memory, where each piece of music is stored as a unique entity characterized especially by verbal labels and knowledge, and by its auditory structure. The third network encompassed several regions, all known to be implied in language processing and to form part of the resting-state auditory network. Music and speech share many similarities, therefore, differences within a language network in experienced musicians seem entirely plausible (and have already been attested). Finally, the fourth network incorporated the right inferior frontal gyrus and the claustrum (bilaterally), structures that belong to the executive control network. We propose that this network could subserve the motor modulation required for the emotionally expressive interpretation of music. At this point, it is important to note that these interpretations are hypothetical, and deserve to be confirmed by behavioral assessments that would be statistically linked to the functional results. Moreover, we cannot provide a causal explanation between brain differences and musical practice, as the present work was cross-sectional, and that some 
studies have suggested brain anatomical (Wong et al. 2007) and functional predisposition for learning (Zatorre et al. 2012; López-Barroso et al. 2013), even at rest (Veroude et al. 2010; Ventura-Campos et al. 2013). In other words, the differences we found in the brain of musically trained individuals might not have been triggered by practice-related plasticity, but might rather have prompted musicians to engage themselves in music and to reach a high level of expertise.

Our supplementary analysis indicated that the functional reorganization we found in the musically experienced group cannot wholly be explained by higher local GM volume. This suggests that other variables (not assessed in this study) are also implicated in the expertise-related resting-state functional reorganization. In future works, it could be worthwhile taking other proxies into account, such as the frequency of training and the number of musical pieces recently learned, in view of the hypothesized role of resting-brain neural activity in consolidating new learning, as well as in maintaining synchronous the activity of areas that are frequently jointly engaged during active states (Fox and Raichle, 2007). Moreover, adding diffusion tensor imaging (DTI) data could help to clarify the link between morphological and resting-state functional modulation of the brain in the framework of expertise-related plasticity given that resting-state functional connectivity also reflects the brain's anatomical connectivity (van den Heuvel et al. 2009). Finally, other sophisticated statistical procedures, such as stochastic dynamic causal modeling (DCM), could provide information about the effective connectivity between functionally connected areas at rest. That means, it could allow having clues regarding the amount and direction of the influence that one area's time series exerts over another.

To conclude, although the results of the present study need to be confirmed and deepened, it suggests that resting-state functional connectivity studied from structurally modified brain areas can contribute to the investigation of expertise-related functional plasticity. In the framework of musical practice, it corroborates that expertise is linked to structural enhancements beyond the sensorimotor cortex. In addition, it also shows that these morphological particularities are accompanied by modulations in their resting-state functional connectivity with many other areas of the brain, ranging from subcortical regions to highlevel cognitive areas, via the primary sensorimotor cortex. 


\section{Acknowledgements}

This study was supported by a French Ministry of Research grant. We would like to thank all the members of the INSERM U1077 team and the staff at the Cyceron center for their support. We are especially grateful to Ms. Leblond and Ms. Mutlu for their help in the redaction and illustration, and to Dr. Lajoie and Dr. Arenaza-Urquijo for their valuable advice during the revision phase. We also thank the two reviewers for their helpful comments. 


\section{References}

Alamy M, Pons JC, Gambarelli D, Trouche E (1995) A defective control of small-amplitude movements in monkeys with globus pallidus lesions: An experimental study on one component of pallidal bradykinesia. Behav Brain Re. 72: 57-62.

Albert NB, Robertson EM, Miall RC (2009) The resting human brain and motor learning. Curr Biol 19:1023-1027.

Anderson BJ (2011) Plasticity of gray matter volume: The cellular and synaptic plasticity that underlies volumetric change. Dev Psychobiol 53:456-465.

Ashburner J, Friston KJ (2005) Unified segmentation. Neuroimage 26: 839-851.

Bangert M, Peschel T, Schlaug G, Rotte M, Drescher D, et al. (2006) Shared networks for auditory and motor processing in professional pianists: Evidence from fMRI conjunction. Neuroimage 30: 917-926.

Bengtsson SL, Nagy Z, Skare S, Forsman L, Forssberg H, Ullén F (2005) Extensive piano practicing has regionally specific effects on white matter development. Nat Neurosci 8(9): 1148-1150.

Bermudez P, Zatorre RJ (2005) Conditional associative memory for musical stimuli in nonmusicians: Implications for absolute pitch. J Neurosci 25: 7718-7723.

Bialystok E, DePape A-M (2009) Musical expertise, bilingualism, and executive functioning. J Exp Psychol Hum Percept Perform 35: 565-574.

Brattico E, Alluri V, Bogert B, Jacobsen T, Vartiainen N, et al. (2011) A functional MRI study of happy and sad emotions in music with and without lyrics. Front Psychology 2: 308. doi:10.3389/fpsyg.2011.00308

Brett M, Anton JL, Valabregue R, Poline J-B (2002) Region of interest analysis using an SPM toolbox. Neuroimage 16, abstract 497.

Crick FC, Koch C. (2005). What is the function of the claustrum? Philos Trans R Soc LordB Biol Sci 360: 1271-1279.

Damoiseaux JS, Rombouts S, Barkhof F, Scheltens P, Stam CJ, et al. (2006) Consistent resting-state networks across healthy subjects. P Natl Acad. Sci 103: 13848-13853.

Dawson WJ (2011) How and why musicians are different from nonmusicians. A bibliographic review. Med Prob Perform Art 26(2): 65-78.

Di X, Zhu S, Jin H, Wang P, Ye Z, et al. (2012) Altered resting brain function and structure in professional badminton players. Brain Connect. 2(4): 225-233.

Dick F, Lee HL, Nusbaum H, Price CJ (2010) Auditory-motor expertise alters "speech selectivity" in professional musicians and actors. Cereb Cortex21: 938-948.

Draganski B, May A (2008) Training-induced structural changes in the adult human brain. Behav Brain Res 192: 137-142.

Duan X, Liao W, Liang D, Qiu L, Gao Q,Liu C, Gong Q, et al. (2012) Large-scale brain networks in board game experts: Insights from a domain-related task and task-free resting state. PloS One 7(3): e32532.

Duncan J, Owen AM (2000) Common regions of the human frontal lobe recruited by diverse cognitive demands. Trends Neurosci 10: 475-483. 
Etkin A, Egner T, Kalisch R (2011) Emotional processing in anterior cingulate and medial prefrontal cortex. Trends Cogn Sci 15: 85-93.

Fauvel B, Groussard M, Eustache F, Desgranges B, Platel H (2013) Neural implementation of musical expertise and cognitive transfers: Could they be promising in the framework of normal cognitive aging? Front Hum Neurosci 7(693): doi: 10.3389/fnhum.2013.00693.

Ford JH, Addis DR, Giovanello KS (2011) Differential neural activity during search of specific and general autobiographical memories elicited by musical cues. Neuropsychologia 49: 2514-2526.

Fox MD, Raichle ME (2007) Spontaneous fluctuations in brain activity observed with functional magnetic resonance imaging. Nat Rev Neurosci 8: 700-711.

Gaab N, Schlaug G (2003) Musicians differ from nonmusicians in brain activation despite performance matching. Ann N Y Acad Sci 999: 385-388.

George MS, Parekh PI, Rosinsky N, Ketter TA, Kimbrell TA, et al. (2012) Understanding emotional prosody activates right hemisphere regions. Arch Neurol 53(7): 665-70.

Gaser C, Schlaug G (2003) Brain structures differ between musicians and non-musicians. J Neurosci 23(27): 9240-9245.

Groussard M, Rauchs G, Landeau B, Viader F, Desgranges B, et al. (2010a) The neural substrates of musical memory revealed by fMRI and two semantic tasks. Neuroimage 53: 1301-1309.

Groussard M, La Joie R, Rauchs G, Landeau B, Chételat G, et al. (2010b) When music and long-term memory interact: Effects of musical expertise on functional and structural plasticity in the hippocampus. PloS One 5(10): e13225.

Hariri AR, Bookheimer SY, Mazziotta JC (2000) Modulating emotional responses: Effects of a neocortical network on the limbic system. Neuroreport 11: 43-48.

Haueisen J, Knösche TR (2001) Involuntary motor activity in pianists evoked by music perception. J Cognitive Neurosci 13: 786-792.

Huijbers W, Pennartz CMA, Rubin DC, Daselaar SM (2011) Imagery and retrieval of auditory and visual information: Neural correlates of successful and unsuccessful performance. Neuropsychologia 49: 1730-1740.

Hyde KL, Lerch J, Norton A, Forgeard M, Winner E, et al. (2009) Musical training shapes structural brain development. J Neurosci 29(10): 3019-3025.

Ilg R, Wohlschlager AM, Gaser C, Liebau Y, Dauner R, et al. (2008) Gray matter increase induced by practice correlates with task-specific activation: A combined functional and morphometric magnetic resonance imaging study. J Neurosc. 28: 4210-4215.

Imfeld A, Oechslin M, Meyer M, Loenneker T, Jancke L (2009)White matter plasticity in the corticospinal tract of musicians: A diffusion tensor imaging study. Neuroimage 46: 600-607.

James CE, Oechslin MS, Van De Ville D, Hauert C-A, Descloux C, Lazeyras F (2013) Musical training intensity yields opposite effects on grey matter density in cognitive versus sensorimotor networks. Brain Struct Funct. doi: 10.1007/s00429-013-0504-z

Jung WH, Kim SN, Lee TY, Jang JH, Choi C-H, et al. (2013) Exploring the brains of Baduk (Go) experts: Gray matter morphometry, resting-state functional connectivity, and graph theoretical analysis. Front Hum Neurosci 7(633).doi: 10.3389/fnhum.2013.00633 
Kristeva R, Chakarov V, Schulte-Möntig J, Spreer J (2003) Activation of cortical areas in music execution and imaging: A high-resolution EEG study. Neuroimage 20:1872-1883.

Kross E, Davidson M, Weber J, Ochsner K. (2009). Coping with emotions past: The neural bases of regulating affect associated with negative autobiographical memories. Bio Psychiatry 65: 361-366.

Lewis CM, Baldassarre A, Committeri G, Romani GL, Corbetta M (2009) Learning sculpts the spontaneous activity of the resting human brain. P Natl Acad Sci 106: 17558-17563.

Liao W, Xu Q, Mantini D, Ding J, Machado-de-Sousa, et al. (2011) Altered gray matter morphometry and resting-state functional and structural connectivity in social anxiety disorder. Brain Res 1388:167-177.

López-Barroso D, Catani M, Ripollés P, Dell'Acqua F, Rodrỉguez-Fornells A, de DiegoBalguer R (2013) Word learning is mediated by the left arcuate fasciculus. PNAS. 110(32): 13168-13173.

Lui S, Deng W, Huang X, Jiang L, Ma X, et al. (2009) Association of cerebral deficits with clinical symptoms in antipsychotic-naive first-episode schizophrenia: An optimized voxelbased morphometry and resting state functional connectivity study. Am J Psychiatry 166: 196-205.

Luo C, Guo Z, Lai Y, Liao W, Liu Q, et al. (2012). Musical training induces functional plasticity in perceptual and motor networks: Insights from resting-state fMRI. PloS One. 7(5): e36568.

Maddock RJ, Garrett AS, Buonocore MH. (2001). Remembering familiar people: The posterior cingulate cortex and autobiographical memory retrieval. Neuroscience. 104: 667676.

Martinelli P, Sperduti M, Piolino P (2012) Neural substrates of the self-memory system: New insights from a meta-analysis. Hum Brain Mapp doi:10.1002/hbm.22008

Mevel K, Landeau B, Fouquet M, La Joie R, Villain N, et al. (2012) Age effect on the default mode network, inner thoughts, and cognitive abilities. Neurobiol Aging 34(4): 1292-1301.

Morris JS, Scott SK, Dolan RJ (1999) Saying it with feeling: Neural responses to emotional vocalizations. Neuropsychologia 37: 1155-1163.

Münte TF, Altenmuller E, Jancke L (2002) The musician's brain as a model of neuroplasticity. Nat Rev Neurosci3: 473-477.

Nakamura K, Kawashima R, Ito K, Sugiura M, Kato T, et al. (1999) Activation of the right inferior frontal cortex during assessment of facial emotion. J Neurophysiol 82: 1610-1614.

Öztürk HA, Tascioglu B, Aktekin M, Kurtoglu Z, Erden I (2002) Morphometric comparison of the human corpus callosum in professional musicians and non-musicians by using in vivo magnetic resonance imaging. J Neuroradial 29: 29-34.

Pallesen KJ, Brattico E, Bailey CJ, Korvenoja A, Koivisto J, et al. (2010) Cognitive control in auditory working memory is enhanced in musicians. PloS One 5(6): e11120.

Pantev C, Herholz SC (2011) Plasticity of the human auditory cortex related to musical training. Neurosci Biobeha Rev 35: 2140-2154.

Payer DE, Baicy K, Lieberman MD, London ED (2012) Overlapping neural substrates between intentional and incidental down-regulation of negative emotions. Emotion 12: 229-235. 
Phan KL, Wager T, Taylor SF, Liberzon I (2002) Functional neuroanatomy of emotion: A meta-analysis of emotion activation studies in PET and fMRI. Neuroimage 16: 331-348.

Platel H, Baron JC, Desgranges B, Bernard F, Eustache F (2003) Semantic and episodic memory of music are subserved by distinct neural networks. Neuroimage 20: 244-256.

Poline JB, Worsley KJ, Holmes AP, Frackowiak RS, Friston KJ (1995) Estimating smoothness in statistical parametric maps: Variability of p values. J Comput Assist Tomogr 19: 788-96.

Price CJ (2012) A review and synthesis of the first 20 years of PET and fMRI studies of heard speech, spoken language and reading. Neuroimage 62: 816-847.

Ross LA, Olson IR (2011) What's unique about unique entities? An fMRI investigation of the semantics of famous faces and landmarks. Cereb Cortex 22: 2005-2015.

Schlaug G, Jäncke L, Huang Y, Staiger JF, Steinmetz H (1995) Increased corpus callosum size in musicians. Neuropsychologia 33: 1047-1055.

Schneider P, Scherg M, Dosch HG, Specht HJ, Gutschalk A, Rupp A (2002). Morphology of Heschl's gyrus reflects enhanced activation in the auditory cortex of musicians. Nat Neurosci 5(7): 688-694.

Schrott LM (1997) Effect of training and environment on brain morphology and behavior. Acta Pædiatr Suppl 422: 45-47.

Seeley WW, Menon V, Schatzberg AF, Keller J, Glover GH, et al. (2007) Dissociable intrinsic connectivity networks for salience processing and executive control. J Neurosci 27(9): 2349-2356.

Sergent J, Zuck E, Terriah S, MacDonald B (1992) Distributed neural network underlying musical sight-reading and keyboard performance. Science 257: 106-109.

Shergill SS, Brammer MJ, Fukuda R, Bullmore E, Amaro E, et al. (2002) Modulation of activity in temporal cortex during generation of inner speech. Hum Brain Mapp 16: 219-227.

Simmons WK, Martin A (2009) The anterior temporal lobes and the functional architecture of semantic memory. J Int Neuropsychol Soc 15(5): 645-649.

Smythies J, Edelstein L, Ramachandran V (2012) Hypotheses relating to the function of the claustrum. Front Integr Neurosci 6: 53-69.

Stoeckel C, Gough PM, Watkins KE, Devlin JT (2009) Supramarginal gyrus involvement in visual word recognition. Cortex 45:1091-1096.

Tambini A, Ketz N, Davachi L (2010) Enhanced brain correlations during rest are related to memory for recent experiences. Neuron 65: 280-290.

Tillmann B, Janata P, Bharucha JJ (2003) Activation of the inferior frontal cortex in musical priming. Cognitive Brain Res 16: 145-161.

Urner M, Schwarzkopf DS, Friston K, Rees G (2013) Early visual learning induces longlasting connectivity changes during rest in the human brain. Neuroimage 77: 148-156.

Vahdat S, Darainy M, Milner TE, Ostry DJ (2011) Functionally specific changes in restingstate sensorimotor networks after motor learning. J Neurosci 31: 16907-16915.

van den Heuvel MP, Hulshoff Pol HE (2010) Exploring the brain network: A review on resting-state fMRI functional connectivity. Eur Neuropsychopharmacol 20: 519-534. 
van den Heuvel MP, Mandl RCW, Kahn RS, Hulshoff Pol HE (2009) Functionally linked resting-state networks reflect the underlying structural connectivity architecture of the human brain. Hum Brain Mapp 30: 3127-3141.

Ventura-Campos N, Sanjuả n A, Gonzả lez J, Palomar-Garcỉa M-A, Rodrỉguez-Pujadas A, et al. (2013) Spontaneous brain activity predicts learning ability of foreign sounds. J Neurosci 33(22): 9295-9305.

Veroude K, Norris DG, Shumskaya E, Gullberg M, Indefrey P (2010) Functional connectivity between brain regions involved in learning words of a new language. Brain Lang. 113: 21-27.

Villain N, Landeau B, Groussard M, Mevel K, Fouquet M, Dayan J, et al. (2010) A simple way to improve anatomical mapping of functional brain imaging. J Neuroimaging 4: 324-333.

Wang Z, Zhang Z, Jiao Q, Liao W, Chen G, et al. (2012) Impairments of thalamic nuclei in idiopathic generalized epilepsy revealed by a study combining morphological and functional connectivity MRI. PloS One 7(7): e39701.

Watkins KE, Strafella AP, Paus T (2003) Seeing and hearing speech excites the motor system involved in speech production. Neuropsychologia 41: 989-994.

Wong PCM, Warrier CM, Penhune VB, Roy AK, Sadehh A, et al. (2007) Volume of left Heschl's gyrus and linguistic pitch learning. Cereb Cortex 18: 828-836.

Yuan K, Qin W, Liu J, Sun J, Liu P, et al. (2010) Gray matter deficits and resting-state abnormalities in abstinent heroin-dependent individuals. Neurosci Lett 482:101-105.

Zatorre RJ, Delhommeau K, Zarate JM (2012) Modulation of auditory cortex response to pitch variation following training with microtonal melodies. Front Psychol 3, 544. doi: 10.3389/fpsyg.2012.00544. 
Table 1

Demographic Characteristics of Musicians and Nonmusicians

\begin{tabular}{ccccccc}
\hline Group & $n$ & $\begin{array}{c}\text { Sex ratio } \\
\text { (male/female) }\end{array}$ & $\begin{array}{c}\text { Age } \\
\text { (years) } \\
\text { mean } \pm \\
S D\end{array}$ & $\begin{array}{c}\text { Education } \\
\text { (years) Mean } \\
\pm \text { SD }\end{array}$ & $\begin{array}{c}\text { Training } \\
\text { duration } \\
\text { (years) } \\
\text { Mean } \pm S D\end{array}$ & $\begin{array}{c}\text { Age at the } \\
\text { start of } \\
\text { practice } \\
\text { Mean } \pm S D\end{array}$ \\
Musicians & 16 & $7 / 9$ & $\begin{array}{c}24.06 \pm \\
3.55\end{array}$ & $14.88 \pm 0.81$ & $16.13 \pm 4.29$ & $7.75 \pm 2.02$ \\
Nonmusicians & 17 & $6 / 11$ & $\begin{array}{c}24.65 \pm \\
3.24\end{array}$ & $15.12 \pm 2.31$ & - & - \\
\hline
\end{tabular}


Table 2

Anatomical Labelling, Cluster Size, MNI Coordinates and Z-value for all analyses (p(unc) $\leq$ $.001, k \geq 15$.

\begin{tabular}{|c|c|c|c|c|c|}
\hline Contrast and anatomical labelling & Cluster size & $\mathbf{x}$ & y & z & Z score \\
\hline \multicolumn{6}{|l|}{ Structural modulated data contrast : Mus-Nonmus } \\
\hline Bilateral posterior cingulate gyrus (BA23) & 83 & 4 & -36 & 34 & 3,78 \\
\hline Right middle cingulate gyrus (BA23) & 28 & 10 & -16 & 42 & 3,70 \\
\hline Left superior temporal gyrus (BA 22) & 15 & -54 & -12 & 4 & 3,70 \\
\hline Right inferior orbitofrontal gyrus (BA11) & 21 & 26 & 38 & -14 & 3,61 \\
\hline \multicolumn{6}{|c|}{ Seed 1 (right posterior cingulate gyrus)-based resting state functional connectivity map contrast : Mus-Nonmus } \\
\hline Right inferior orbitofrontal gyrus (BA11) & 39 & 8 & 32 & -14 & 3,79 \\
\hline \multicolumn{6}{|c|}{ Seed 2 (right middle cingulate gyrus)-based resting state functional connectivity map contrast : Mus-Nonmus } \\
\hline Right inferior frontal gyrus (BA47) & 15 & 42 & 34 & 0 & 3,69 \\
\hline Left superior temporal pole (BA38) & 27 & -42 & 12 & -20 & 3,5 \\
\hline \multicolumn{6}{|c|}{ Seed 3 (left temporal superior gyrus)-based resting state functional connectivity map contrast : Mus-Nonmus } \\
\hline Left premotor cortex (BA8) & 88 & -22 & 12 & 56 & 3,98 \\
\hline Right globus pallidus & 18 & 12 & 0 & -14 & 3,78 \\
\hline \multirow{3}{*}{ Bilateral supramarginal gyrus (BA39/40) } & $121 / 33$ & $-50 /-30$ & $-50 /-58$ & $38 / 42$ & $3,72 / 3,42$ \\
\hline & & & & & \\
\hline & 73 & 58 & -50 & 22 & 3,46 \\
\hline \multirow{2}{*}{$\begin{array}{l}\text { Bilateral inferior part of the precentral } \\
\text { gyrys (BA48) }\end{array}$} & 31 & -48 & 4 & 4 & 3,55 \\
\hline & $61 / 18$ & $34 / 50$ & $-20 / 0$ & $52 / 6$ & $3,54 / 3,40$ \\
\hline Left middle anterior temporal gyrus (BA20) & 33 & -48 & 2 & -34 & 3,42 \\
\hline Left middle frontal gyrus (BA45) & 16 & -44 & 36 & 26 & 3,18 \\
\hline \multicolumn{6}{|c|}{ Seed 4 (right inferior frontal gyrus)-based resting state functional connectivity map contrast : Mus-Nonmus } \\
\hline \multirow{3}{*}{ Bilateral claustrum } & 64 & -26 & 24 & 10 & 3,41 \\
\hline & & & & & \\
\hline & 19 & 38 & 18 & 0 & 3,36 \\
\hline
\end{tabular}


Click here to download high resolution image

Musicians

Seed 1 : Right posterior cingulate gyrus
Nonmusicians

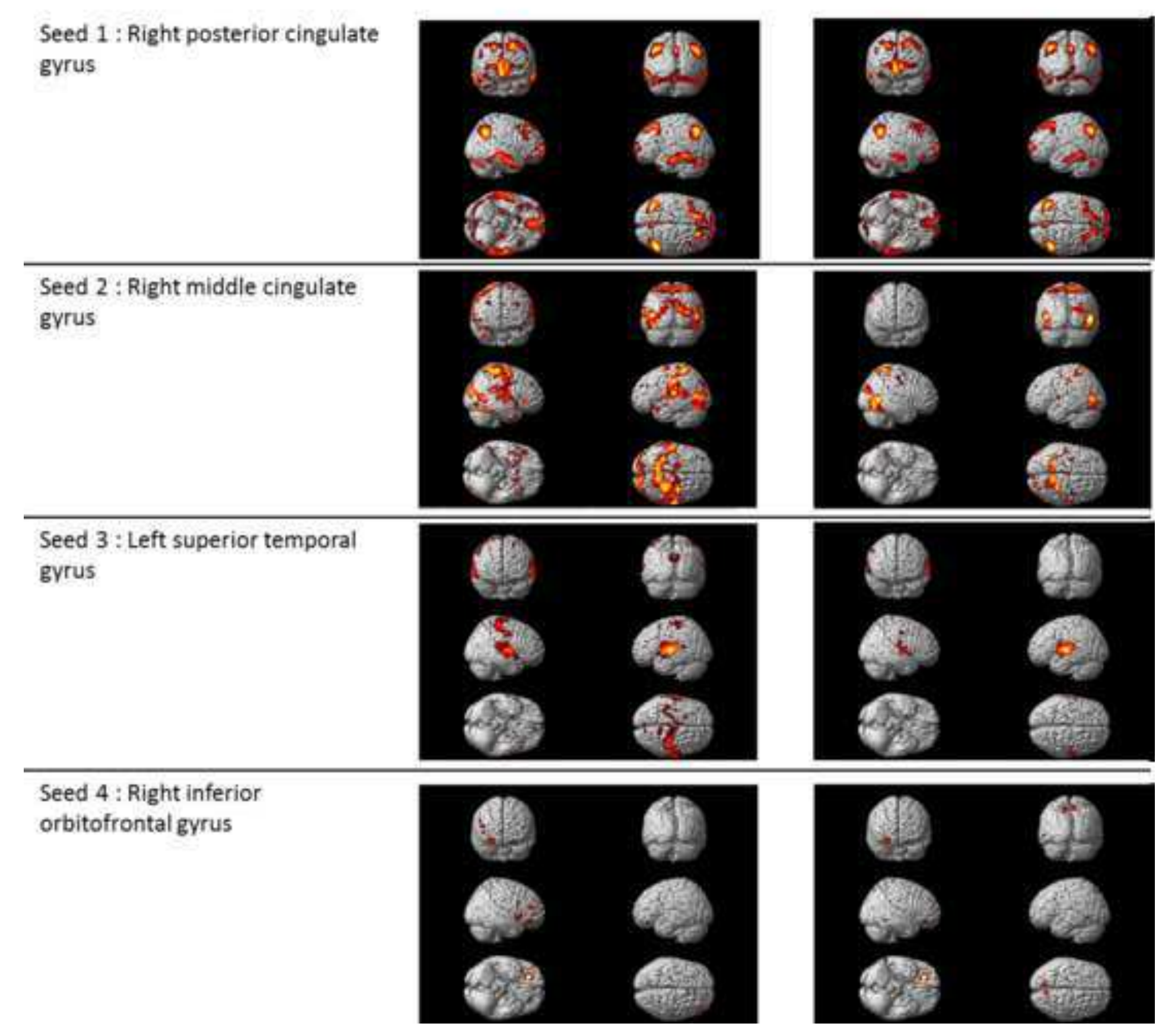




\section{Figure 1}

Click here to download 9. Figure: Figure1_caption.docx

Figure1. Image showing the four resting-states functional connectivity maps for musicallyexperienced group and nonmusicians group. $p$ (fwe) $\leq .0001$ 
Click here to download high resolution image

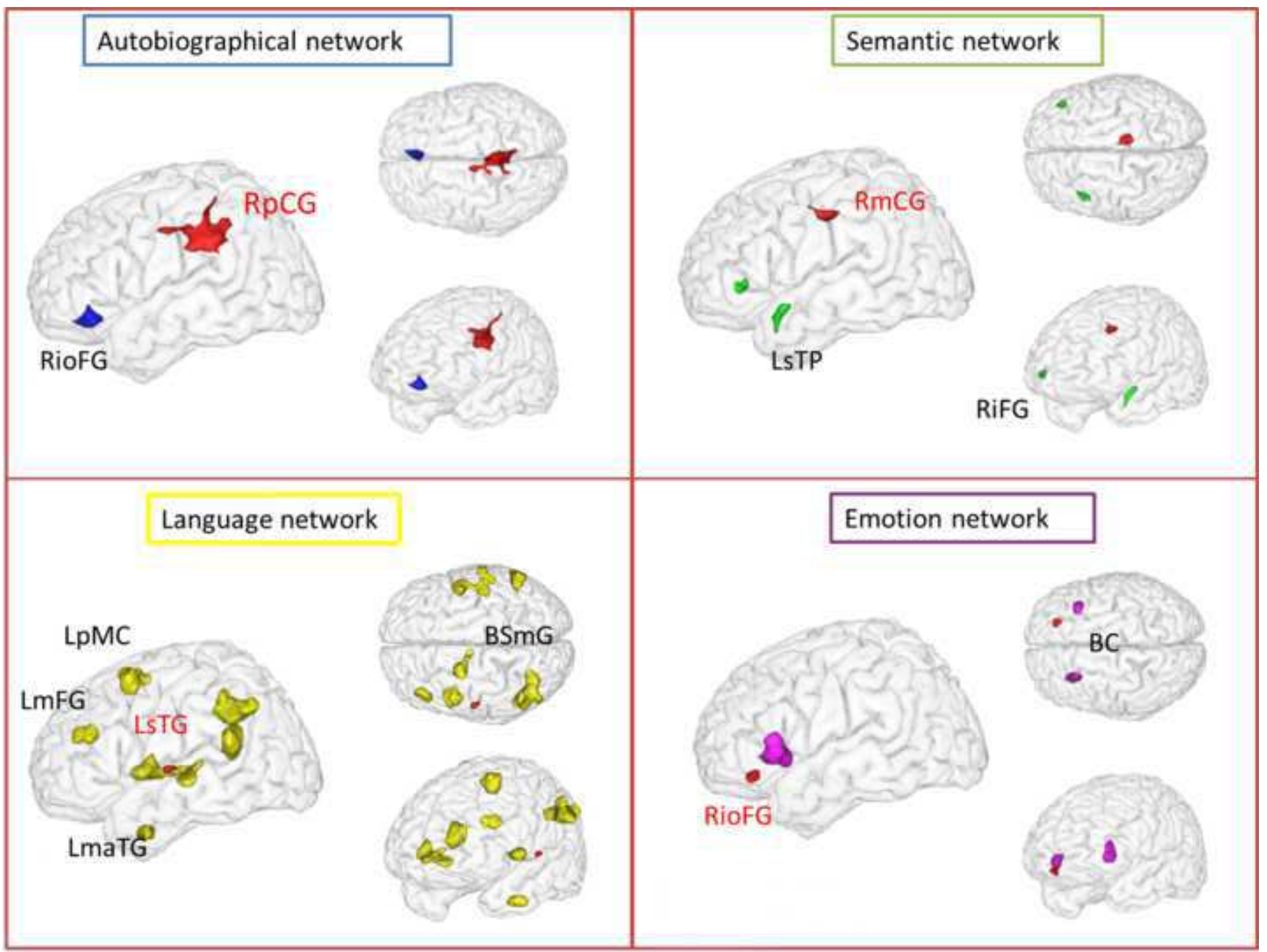


Figure 2. Image showing the four clusters of enhanced gray matter volume in the musician group (red regions), as well as the brain areas with resting-state time series more correlated in the musician group (shown in blue, green, yellow, and purple). $p$ (unc) $\leq .005$.

RpCG: Right posterior Cingulate Gyrus; RmCG: Right middle Cingulate Gyrus; RioFG: Right inferior orbitoFrontal Gyrus; LsTG: Left superior Temporal Gyrus; LsTP: Left superior Temporal Pole; RiFG: Right inferior Frontal Gyrus; BC: Bilateral Claustrum; BSmG: Bilateral Supramarginal Gyrus; LmaTG: Left middle anterior Temporal Gyrus; LmFG: Left middle Frontal Gyrus; LpMC: Left preMotor Cortex. 
Click here to download 9. Figure: Figure 3_caption.docx

Figure 3. Graph displaying averaged targets' correlation Z-values for musically-experienced group and nonmusicians group, and indicating whether these values are significantly different than 0 $\left({ }^{*} p \leq 0.05, * * p \leq 0.01, * * * p \leq 0.001\right)$. 


\section{Click here to download high resolution image}

\section{Seed1: Right posterior cingulate gyrus} $Z$ values

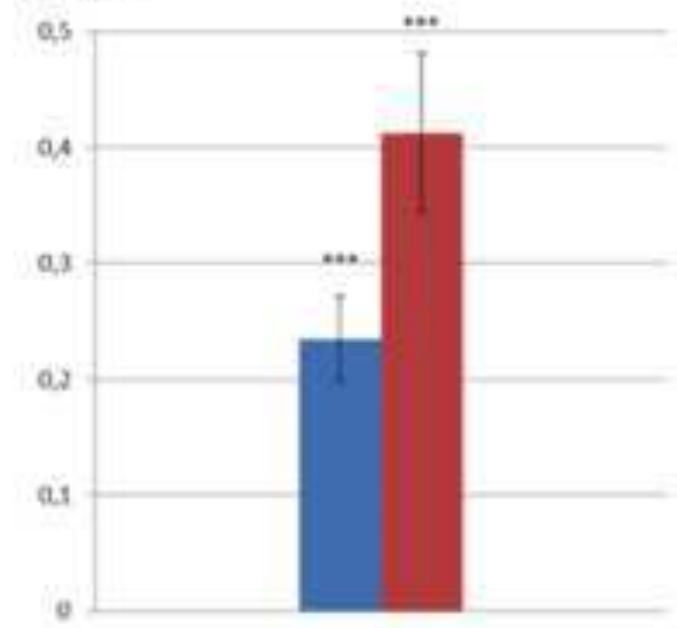

Rieht inferior orbitofrontal avrus
Seed2: Right middle cingulate gyrus

$Z$ values

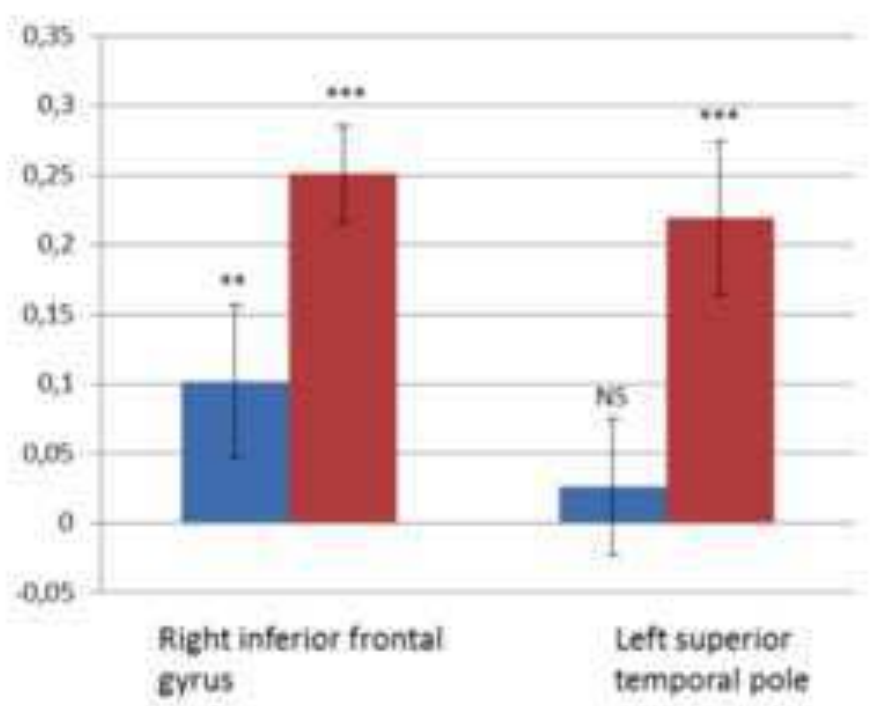

\section{Seed4: Right inferior orbitofrontal gyrus}

\section{$Z$ values}

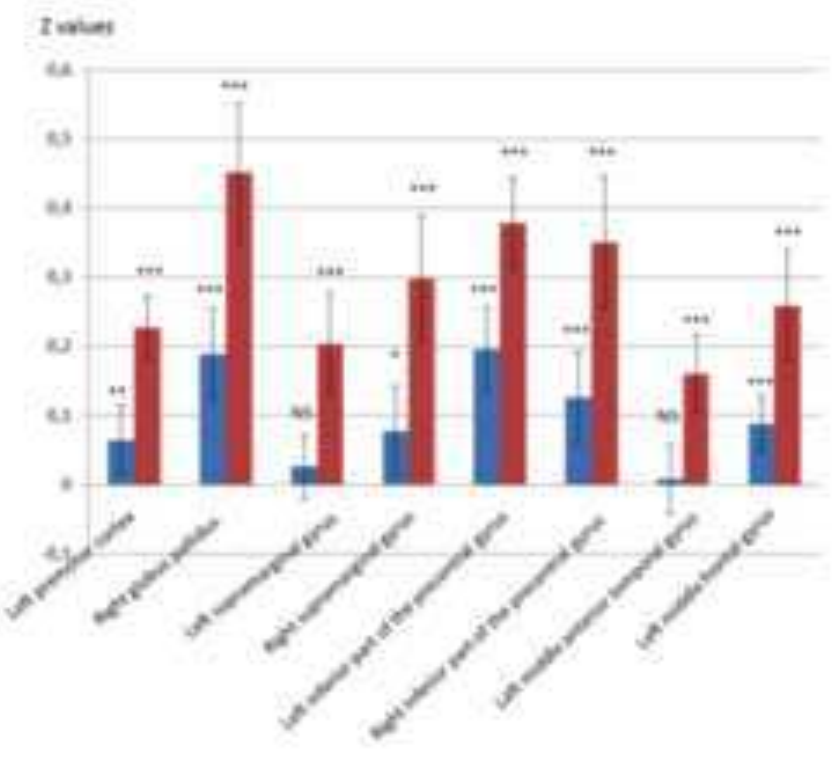

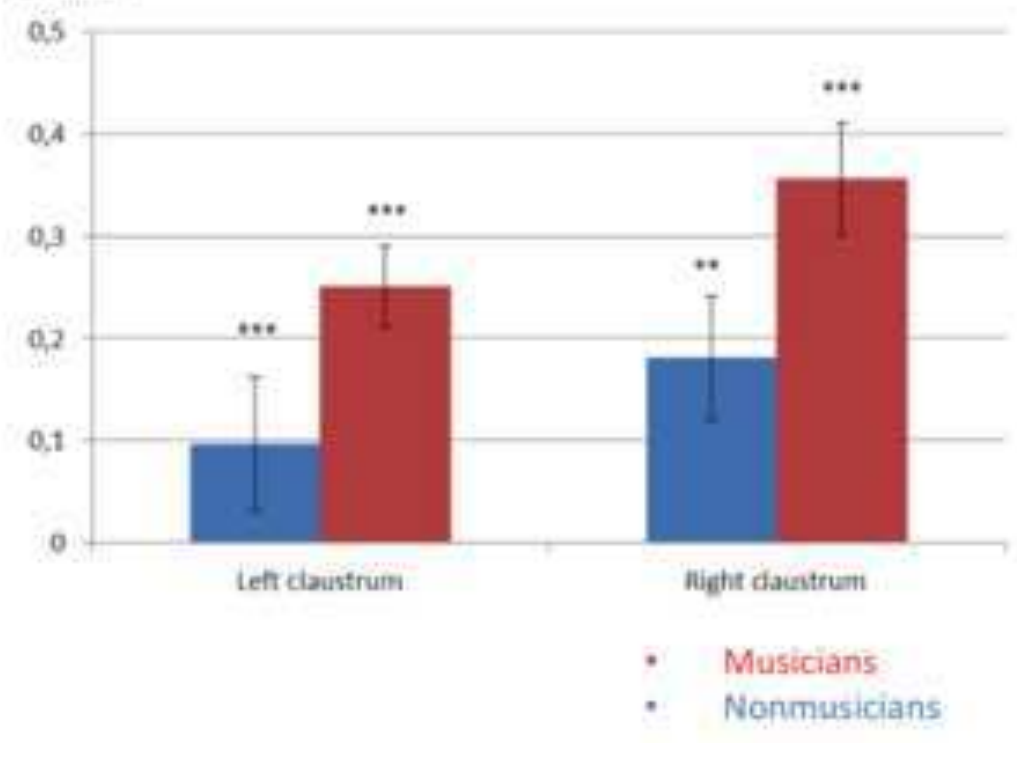

